\title{
ON THE VARIABILITY OF THE CENTRAL STAR OF THE PLANETARY NEBULA $\mathrm{H}_{\mathrm{z}}$ 1-5
}

\author{
G. H. HeRBIG \\ (Lick Observatory, U.S.A.) \\ and \\ A. A. BOYARCHUK \\ (Crimean Astrophysical Observatory, \\ U.S.S.R.)
}

During 1960-67 we have studied the variable star FG Sge=377.1943 Sge, which is located exactly in the centre of an image of the planetary nebula

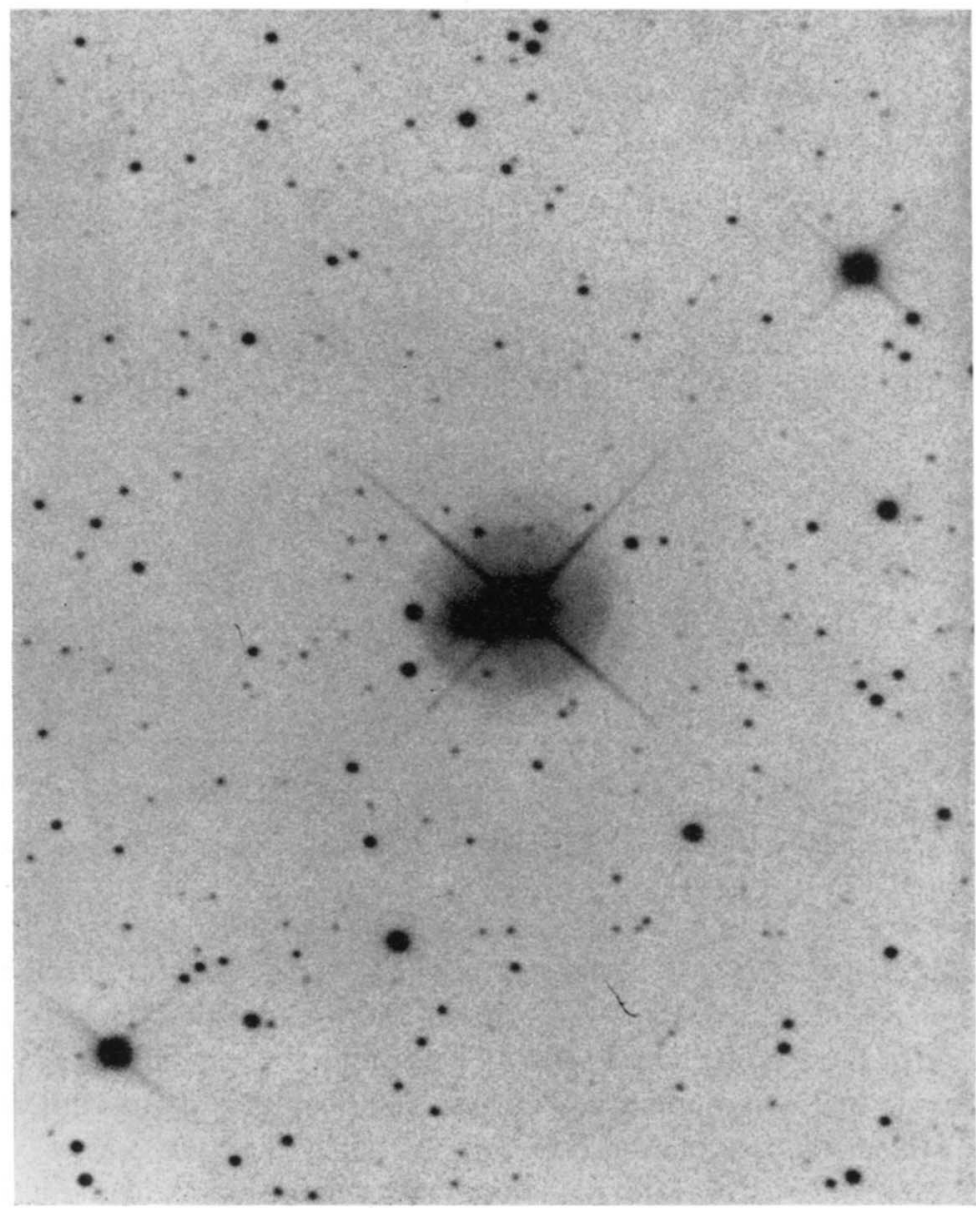

FIG. 1. The nebulosity surrounding FG Sge.

Osterbrock and O'Dell (eds.), Planetary Nebulae, 383-385. (c) I.A.U. 
$\mathrm{H}_{z}$ 1-5, as shown on Figure 1. The most significant feature of the central star is the continuous increase of its brightness during the past 70 years, as one can see from Figure 2. During the same time, the temperature of the central star has apparently decreased, although direct information to this effect is available only since 1955. In 1955 the star had spectral type B4, while by 1967 it had become A5. The observational data can be explained by the continuous ejection of gas from the central star, which began at the end of the preceding century. The central star at that time must have had $T_{*} \approx 63300^{\circ} \mathrm{K}$ and $M_{\mathrm{pg}} \approx+1 \cdot 3$, on this model.

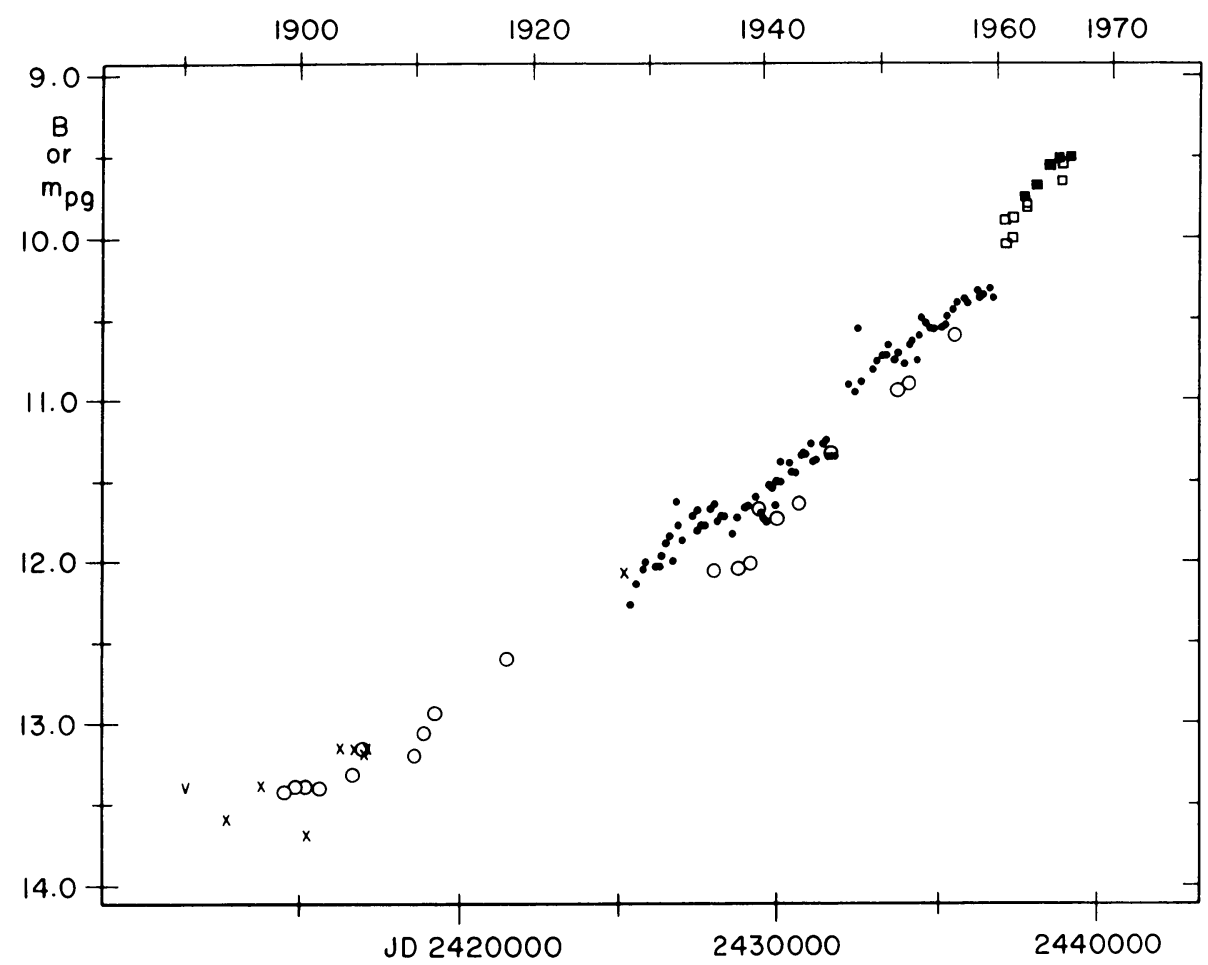

FIG. 2. The blue magnitudes of FG Sge from the beginning of the 20th century.

Those values agree very well with those of central stars of planetary nebulae. The expansion velocity of the new shell will, in about 3000 years, cause the new envelope to reach the size of the existing nebula $\mathrm{H}_{\mathrm{z}} 1-5$. Therefore, it is possible to produce more than one nebula by the same central star. Another possibility is that this central star is a double system, whose components have practically the same masses.

In any case, we believe that future investigations of this object will give us important information about the formation and evolution of planetary nebulae. 


\section{DISCUSSION}

Münch: What kind of a spectrum does this most remarkable star have?

Boyarchuk: The spectral type in 1955 was B4 I with P Cyg features, but in 1967 it was A5 Ia without any P Cyg characteristics.

Kohoutek: I am able to confirm Boyarchuk and Herbig's result concerning the planetary $\mathrm{H}_{\mathrm{z}}$ 1-5. Our photoelectric observations with the $65-\mathrm{cm}$ reflector at Ondřejov between 1965-67 show an increase of the star brightness of about $\mathbf{0 . 2} \mathrm{mag}$. Also, the spectrum of the star in September 1965 from Asiago shows no trace of emission lines, so that perhaps the nebula no longer radiates. 